\title{
Two extensions of the anchor-range effect
}

\author{
DONALD L. KING and MOHAMMAD-KAZEM ATEF-VAHID \\ Howard University, Washington, D. C.
}

\begin{abstract}
A stimulus from one category increased the rated similarity of two stimuli from another category. In addition, "different" responses, but not "same" responses, to same-category stimuli became slower when they occurred in blocks of trials with a stimulus from a second category. Another experiment employed figural stimuli in which two small disks and a large or very large (extreme) disk were simultaneously present. The extreme disk led to slower "different" and faster "same" responses to the two small disks. Slow "different" and fast "same" responses may indicate high perceived similarity. Consequently, both the second-category stimulus and the extreme disk, although logically irrelevant, may have increased the perceived similarity of more related stimuli. It is concluded that the second-category stimuli and the extreme disk functioned as conventional anchors. The results also call into question memorial and criterial variance theories and support category-superordinate and distance-density theories of the anchor-range effect.
\end{abstract}

An anchor (extreme) stimulus impairs the absolute identification of more related stimuli that vary along the same dimension. For example, Gravetter and Lockhead (1973) found that a relatively quiet third sound impaired the identification of two louder sounds. The detrimental influence of a large stimulus range on identification that was discovered by Pollack (1952) can be similarly interpreted (Gravetter \& Lockhead, 1973). Identification of the anchor or end stimuli is, for the most part, unaffected (e.g., Pollack, 1952).

A reasonable proposition is that an anchor stimulus increases the perceived similarity of the more related stimuli. This increase would be responsible for the identification impairment. Moreover, this increase-inperceived-similarity position is also supported by category rating (Bevan \& Pritchard, 1963), magnitude estimation (DiLollo \& Kirkham, 1969), and speeded "same" and "different" response results (King, 1983).

The anchor and more related stimuli have generally occurred successively. Therefore, it appears that memory for the anchor is causal.

The increase-in-perceived-similarity interpretation has received relatively little attention, possibly because the use of absolute identification, rating, and estimation measures has fostered memorial and judgmental interpretations. A memorial proposal is that the anchor or end stimuli are more accurately identified because they are

This research was supported by National Institute of Mental Health Grant 5-T-34-MH16580-03. The authors wish to thank Michael E. Barnes and Marcia E. Sutherland for help in data collection. Please send requests for reprints to Donald L. King, Department of Psychology, Howard University, Washington, DC 20059.

Experiments 2 and 3 were reported at the Eastern Psychological Association meeting in New York, April 1981. Experiment 4 was reported at the American Psychological Association meeting in Los Angeles, August 1981. Papers on Experiments 1 and 5 were delivered at the Eastern Psychological Association meetings in Baltimore, April 1982 and April 1984, respectively. rehearsed more, with identification consequently deteriorating as the distance from the anchors increases (Eriksen \& Hake, 1957). It is also known that absolute identification can be impaired by factors known to facilitate forgetting (Siegel, 1972).

Criterial variance theories (Durlach \& Braida, 1969; Gravetter \& Lockhead, 1973) are based on Thurstone's approach to categorical judgment (Torgerson, 1958). They therefore assume that the sensory influence of a stimulus is unaltered, the stimulus belongs to a category that corresponds to a subrange of a dimension, responding is based on category membership, and the anchor affects category membership and hence memorial or judgmental (nonperceptual) processes. Durlach and Braida assumed that the sensation is compared to the "context of sensations determined from previous signals"' (p. 376) and that the distribution of the resulting numerical representation increases with the width (range) of the context. Gravetter and Lockhead assumed that an increase in range increases the width of the boundaries of the judgmental categories.

An anchor-like result, the ability of a stimulus from a second category to increase the rated similarity of stimuli from the same category, has also been reported (Sjöberg, 1972; Tversky, 1977). Tversky proposed that the secondcategory stimulus increases the diagnosticity (a type of salience) of the features of the stimuli that are unique to the other category. In addition, this increase in diagnosticity results in the increase in rated similarity.

Nevertheless, categories may be involved regardless of whether or not features are directly responsible for the increase in rated similarity. For example, the secondcategory stimulus may increase the strength of the category (superordinate) for the first-category stimuli, and this increase may augment rated similarity directly (cf. Lockhead, 1972).

This category-superordinate theory applies to the anchor-range effect. For example, it assumes that a soft 
sound increases the perceived similarity of two loud sounds because it increases the apprehension of a category of loud sounds or affects some correlated factor (e.g., diagnosticity). The soft sound itself would be perceived as a second (different) event. In general, the theory maintains that (1) the perceived similarity among stimuli increases with the strength of the category (superordinate) to which they contribute, (2) the anchor increases the strength of the category-superordinate to which the more related stimuli contribute, and (3) the anchor establishes its own (singleton) event.

Krumhansl (1978) accounted for the anchor-range effect with her distance-density theory. Its core assumption is that "two points in a relatively dense region of a stimulus space would have a smaller similarity measure than two points of equal interpoint distance but located in a less dense region of the space" (p. 446). Density is related to measures of physical similarity (e.g., Krumhansl, 1978, p. 446). Thus, two stimuli are in a higher density surround when the third stimulus is similar to these two stimuli than when it is an anchor. The core assumption then predicts that the two stimuli will be perceived as less similar with a similar third stimulus than with an anchor. Thus, the anchor-range effect is interpreted as an increase-in-perceived-similarity outcome and its explanation follows directly from the core assumption.

One purpose of the present research was to obtain evidence that the anchor-range effect occurs in two nonconventional situations. A second was to argue against the rehearsal, forgetting, and criterial variance theories. A third was to more directly support the increase-inperceived-similarity position, and thereby the categorysuperordinate and distance-density theories as well.

Experiments 1-4 employed two stimuli from one category and a third stimulus (the anchor) from a second category. The stimuli belonged to relatively dissimilar categories. They would therefore have positions on a number of unknown dimensions, whereas the criterial variance theories were designed for a single known dimension. Experiment 5 employed figural stimuli in which two small and one large or very large (i.e., anchor) disk occurred simultaneously. A positive result due to a simultaneously present anchor would rule out memorial explanations. In addition, the use of figural (perceptual) stimuli would be consistent with the increase-in-perceivedsimilarity (and hence perceptual) interpretation.

Experiment 1 employed similarity ratings. The secondand same-category stimuli were simultaneously present, and therefore rehearsal and forgetting were not influential. Experiments 2-5 used speeded "same" and "different" responses to simultaneously present stimuli to measure perceived similarity. Slow cognitive (nonperceptual) influences that operate after stimulus onset were thereby ruled out. Furthermore, because the task involved comparing simultaneously present stimuli, the rehearsal and forgetting possibilities became less likely. The criterial variance theories were questioned because they assume that responding is based on judgmental categories rather than on a direct comparison between simultaneously present stimuli.

\section{EXPERIMENT 1}

The categories employed in the previously mentioned research of Sjöberg (1972) and Tversky (1977) belonged to the same superordinate category. For example, Tversky used American and European countries. Our Experiment 1 used stimuli from less related categories. If an anchor-range result could be obtained with stimuli from relatively dissimilar categories, the stimuli would have to have positions on multiple dimensions that would be relatively difficult to specify, thereby making the criterial variance theories less plausible. The stimuli from the same and second categories appeared on the same page in order to eliminate rehearsal and forgetting. A relatively dissimilar second-category stimulus may still increase the apprehension of the first category. In addition, it results in a low density in the area of the first-category stimuli. Therefore, the category-superordinate and distance-density theories predicted that the relatively dissimilar secondcategory stimuli would continue to yield an increase-inperceived-similarity result.

\section{Method}

Stimuli, Design, and Subjects. The same-category stimuli consisted of three words, patterns, and nonsense syllables. The A, B, and $\mathrm{C}$ items were HAT, BUS, and DOG, respectively,

$$
*_{* *}^{* *},{ }^{*} * * \text {, and }{ }^{*} *^{* * *} \text {, }
$$

respectively, and CEF, TOV, and GAH, respectively. Two-digit numbers were the second-category stimuli.

The same-category group of subjects received $A B, A C$, and $B C$ pairs that were formed from the three items of each category. The second-category group received a two-digit number in place of each of the C items, for instance, 27 in place of DOG.

There were 28 Howard University undergraduates in each of these two independent groups.

Procedure. The subjects were tested during classes. Each subject received a four-page booklet. Page 1 contained written instructions, an illustrative pair, and a copy of the rating scale. The instructions included the information that a rating of 0 should mean that there was absolutely no relation between the two items of a pair and that a rating of 100 should mean that the two items were identical. The subjects were not instructed about the type of similarity on which they were to base their ratings.

The words, patterns, and nonsense syllables appeared on pages 2,3 , and 4 , respectively. The stimuli were typed in pica. The members of a pair appeared side by side. Adjacent to each pair was a line that was calibrated from 0 to 100 ; the subjects marked this line to indicate their similarity ratings.

\section{Results}

Both groups rated the $\mathrm{AB}$ (e.g., HAT-BUS) pairs. The similarity ratings for these pairs were analyzed with a 2 (group: second- and same-category) $\times 3$ (stimuli: words, 
patterns, and nonsense syllables) factorial analysis of variance (ANOVA). The second factor was within subject.

The similarity ratings for the second- and same-category groups were 48 and 28 , respectively $[F(1,38)=9.08$, $p<.01]$. In other words, two same-category stimuli were rated as more similar when a number was the third stimulus. The interaction was not significant $[F(2,76)=$ $0.61]$.

\section{Discussion}

Experiment 1 extends Sjöberg's (1972) and Tversky's (1977) results by showing that a stimulus from a relatively different second category can also increase the rated similarity of two same-category stimuli.

The dimensions for the experiment's same- and secondcategory stimuli are difficult to specify, in opposition to the criterial variance theories.

The same- and different-category stimuli remained present while the ratings were made, and therefore rehearsal and forgetting were not involved.

The category-superordinate theory applies. For example, 27 may have increased the apprehension of a category that HAT and BUS establish, for instance, the category of printed words. The increase in the rated similarity of HAT and BUS follows.

The two target same-category stimuli should have been located in a less dense region when the third stimulus was a member of another category, and hence the distancedensity theory also predicts the obtained results.

\section{EXPERIMENT 2}

Similarity ratings are probably influenced by slow cognitive (nonperceptual) strategies that operate after stimulus onset (Shepard, 1964). However, slow postonset processes cannot affect a current speeded response. Furthermore, we thought that speeded "different" responses could be used to measure perceived similarity, because there is evidence that more similar stimuli result in slower "different" responses (Eriksen, O'Hara, \& Eriksen, 1982; Hawkins \& Shigley, 1972; Krueger, 1973; Proctor, 1981). In addition, perceived, rather than physical, similarity is influential (Watson, 1981).

The stimuli were HAT, BUS, DOG, and 27, as in Experiment 1 . Blocks of trials with pairs formed from the set of all three words alternated with blocks of trials with pairs formed from HAT, BUS, and 27. These blocks are called the all-words blocks and 27 blocks, respectively. We thought that the memory for 27 would increase the perceived similarity of HAT and BUS. Consequently, we predicted that responding to the HAT-BUS pair would be slower when it occurred in the 27 blocks than when it occurred in the all-words blocks.

The members of the pairs occurred simultaneously and therefore could be directly compared. Consequently, rehearsal of the anchor-like 27 was probably not facilita- tive. Moreover, this rehearsal could even have slowed responding to pairs that did not include 27 . In addition, direct comparison would appear to minimize an influence of forgetting.

The Thurstonian criterial variance theories assume that the response is based on the judgmental category to which an individual stimulus belongs. Presumably, they would therefore assume that responding is based on a comparison between the judgmental categories for individual stimuli, rather than on a comparison between the actual stimuli. One might question how understanding is furthered by the assumption that categories, rather than immediately present stimuli, are compared.

The speeded response measure of perceived similarity indicates how quickly a change in perceived similarity can occur, and hence indicates the time required for any theoretical process to operate. Therefore, according to the category-superordinate theory, a positive outcome would suggest that a category or correlated factor can quickly affect perceived similarity, even though the category's strength is modified memorially. According to the distance-density theory, a positive outcome would suggest that density can quickly affect perceived similarity.

\section{Method}

Design and trial considerations. The 27 blocks contained the following six pairs: 27-27, HAT-HAT, BUS-BUS, 27-HAT, BUS27, and HAT-BUS. The all-words blocks contained these six pairs: DOG-DOG, HAT-HAT, BUS-BUS, DOG-HAT, BUS-DOG, and HAT-BUS. Thus, the two sets of pairs were identical, except that 27 and DOG were exchanged.

There were 30 trials in each block. The six pairs of each block occurred once every 6 trials. Thus, there were five repetitions of each pair within each block. The serial order of the six pairs of each group of 6 trials was determined randomly, with one exception: the first trial of a block always began with a 27-27 or DOGDOG pair. The intent was to have incremented the memory for 27 and DOG by the time the first HAT-BUS trial occurred.

Both the 27 and all-words blocks occurred twice. ABAB and BABA orders were used. The identical and corresponding pairs of Blocks 1 and 2 and Blocks 3 and 4 occurred in the same sequence. For example, if the HAT-BUS pair occurred on Trial 15 of Block 1, it also occurred on Trial 15 of Block 2.

Stimuli. HAT, BUS, DOG, and 27 were typed in pica. Uppercase letters and Arabic numerals were used. The two stimuli of a pair appeared to the left and right of the centers of $7.6 \times 12.7 \mathrm{~cm}$ $(3 \times 5$ in.) cards. The characters for each of these stimuli were in adjacent typewriter spaces, and the two stimuli were separated by three typewriter spaces. The width of a single space was about $2.5 \mathrm{~mm}$, and the height of the characters was about $3 \mathrm{~mm}$.

Apparatus, Procedure, and Subjects. A Gerbrands T2BC twofield tachistoscope was used. One arm contained a warning stimulus, a blank $7.6 \times 12.7 \mathrm{~cm}$ card. The second arm contained the cards with the pairs. The distance from the cards to the cornea was approximately $59 \mathrm{~cm}$.

Subjects were instructed to respond only to different pairs. The experimenter initiated a trial by saying "Ready." Then he presented the warning stimulus for $1 \mathrm{sec}$. A 1-sec period of darkness followed. Then a pair occurred. Responding was manual. The timing for the stimuli and responding was to the nearest $10 \mathrm{msec}$. The experimenter terminated a pair directly after the subject's response or $3 \mathrm{sec}$ after its onset. The experimenter told the subjects whether they were correct and how fast they responded. 
The subjects were 6 Howard University undergraduates unfamiliar with the purpose of the research. Three subjects received the ABAB order of blocks and 3 the BABA order.

\section{Results}

The reaction times to individual pairs were the entries for the ANOVA. It was a 2 (order: ABAB and BABA) $\times 2$ (type of block: 27 and all-words) $\times 3$ (type of pair) $\times 2$ (block repetition) $\times 5$ (pair repetition) factorial. The first factor was between subjects.

The important result was that the "different" response time means for the HAT-BUS pair were 720 and $637 \mathrm{msec}$ for the 27 and all-words blocks, respectively $[F(1,8)=$ $7.85, p<.05$, planned-comparisons test]. The other significant outcomes are attributable to practice or to nonidentical pairs (e.g., the 27-HAT and DOG-HAT pairs only corresponded), so they need not be considered.

The mean proportion of errors (false "differents") to the three same pairs in a block was .072 for both the 27 and all-words blocks.

\section{Discussion}

The error proportions argue that the slower "different" responses to the HAT-BUS pair on the 27 blocks were not due to a criterion shift between the 27 and all-words blocks. The introduction indicated that slow "different" responses can be considered to indicate high perceived similarity. Therefore, HAT and BUS may have been perceived as more similar when they occurred in the 27 blocks. Consequently, memory for the anchor-like $27 \mathrm{~s}$ of the 27-27, BUS-27, and 27-HAT pairs may have increased the perceived similarity of HAT and BUS. Experiment 4 argues against the possibility that the 27 blocks were simply more difficult.

Slow cognitive (nonperceptual) processes occurring after stimulus onset may affect a similarity rating as it is being made. However, a fast response cannot be affected in this way. Therefore, the present experiment furthers the possibility that the second-category influence on similarity is perceptual.

There was no discernible advantage to rehearsing 27 , because the task was to discriminate between simultaneously present stimuli, and because half the pairs in the 27 blocks did not contain a 27 . That forgetting was causal also seems unlikely, because stimuli did not have to be discriminated from related stimuli in memory. The memorially based theory of Durlach and Braida (1969) is similarly questioned.

Perceptual discriminations between simultaneously present stimuli have not been generally considered to be made by comparing judgmental categories, and therefore the Thurstonian criterial variance theories are questioned.

According to the category-superordinate theory, the results of Experiment 2 imply that a HAT-BUS superordinate or a correlated factor was able to quickly affect the perceived similarity of HAT and BUS, even though the memory for 27 was also influential. According to the distance-density theory, it is density that is capable of quickly increasing perceived similarity.

\section{EXPERIMENT 3}

The word stimuli of Experiment 2 were replaced by patterns formed from asterisks. The dimensions assumed by the criterial variance theories become more problematical when stimuli from less similar categories are employed. Replication would also indicate that the speeded response results could be obtained with nonalphanumeric items as the same-category stimuli.

The four-asterisk (4ASTERISK), five-asterisk (5ASTERISK), and six-asterisk (6ASTERISK) stimuli were

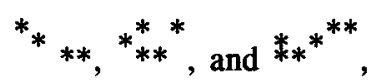

respectively. They corresponded to the DOG, HAT, and BUS stimuli, respectively, of Experiment 2. There were 5 subjects in each group. Otherwise, the method of Experiment 2 was followed exactly.

The ANOVA for Experiment 2 was repeated. The 5ASTERISK-6ASTERISK pair corresponds to the critical HAT-BUS pair of Experiment 2. The mean response times to this pair were 799 and $746 \mathrm{msec}$ for the 27 and all-asterisks blocks, respectively $[F(1,16)=5.63$, $p<.05$, planned-comparisons test]. The mean proportion of errors (false "differents") equaled .077 for both the 27 and all-asterisks blocks.

The results of Experiment 2 were replicated. The analogous conclusion is that the memory for 27 increased the perceived similarity of the 5ASTERISK and 6ASTERISK stimuli. The dimensions for the number and pattern stimuli are problematical, in opposition to the criterial variance theories. Experiment 3 also extended the results of Experiment 2 to nonalphanumeric same-category stimuli.

\section{EXPERIMENTS 4A AND 4B}

In Experiment 4, we sought evidence against the possibility that the slower "different" responses to samecategory pairs were due to the greater difficulty of the 27 blocks (e.g., because of a need to switch attention between categories). Experiment 4A was essentially identical to Experiments 2 and 3. However, in Experiment $4 \mathrm{~B}$, subjects were instructed to respond to same pairs rather than to different pairs. The purpose was to show that slower "same" responses to the analogous pairs would not occur and thereby to argue that the 27 blocks were not more difficult.

Furthermore, the occurrence of faster "same" responses to the same-category stimuli in the 27 blocks would additionally support the increase-in-perceivedsimilarity interpretation. This is because more similar stimuli result in faster "same" responses (Eriksen et al., 1982; Hawkins \& Shigley, 1972; Krueger, 1973; Proc- 
tor, 1981), and because perceived rather than physical similarity is influential (Watson, 1981).

The expected "same" and "different" response results would add to the arguments against the rehearsal and forgetting theories, because it is unlikely that rehearsal and forgetting would differentially affect these responses.

The expected results would also additionally argue against the criterial variance theories. The problem is that it is unclear how the anchor's influence on judgmental categories accounts for a differential effect on "same" and "different" responses.

Stimuli that result in relatively fast responding were used in order to suggest that the increase-in-perceivedsimilarity effect can occur relatively quickly.

\section{Method}

A square, circle, and triangle replaced the HAT, BUS, and DOG stimuli, respectively. These forms were obtained from Letraset Sheet 2453. They were solid black, and the length of their sides (square and triangle) or diameter (circle) was $7 \mathrm{~mm}$. The triangle and square were upright. The forms were placed so that their centers were $7.5 \mathrm{~mm}$ from the center of the cards on which they were placed.

The instructions were to respond to different pairs in Experiment $4 \mathrm{~A}$ and to same pairs in Experiment $4 \mathrm{~B}$. There were 6 subjects in Experiment $4 \mathrm{~A}$ and 10 different subjects in Experiment $4 \mathrm{~B}$. Otherwise, the two experiments were identical, and the method of Experiment 2 was followed exactly.

\section{Results}

The data from Experiments $4 \mathrm{~A}$ and $4 \mathrm{~B}$ were combined for the ANOVA. Only the response times to the target pairs, that is, the same-category pairs that occurred in both the 27 and all-forms blocks, were analyzed. For Experiment $4 \mathrm{~A}$, the average of the response times to the five SQUARE-CIRCLE pairs that occurred within each block was the entry for the ANOVA. For Experiment 4B, the average of the response times to the five SQUARESQUARE and five CIRCLE-CIRCLE pairs that occurred within each block was the entry. The ANOVA became a 2 (type of response: "same" and "different") $\times 2$ (order: ABAB and BABA) $\times 2$ (type of block: 27 and all-forms) $\times 2$ (block repetition) factorial. The last two factors were within subject.

The predicted interaction between the type of response and type of block factors occurred $[F(1,12)=8.02$, $p<.05]$. The mean response times to the SQUARECIRCLE pair in the 27 and all-forms blocks were 484 and $451 \mathrm{msec}$, respectively. However, the mean response times to the SQUARE-SQUARE and CIRCLE-CIRCLE pairs in the 27 and all-forms blocks were 419 and $423 \mathrm{msec}$, respectively. A simple effects test revealed that the slower responding to the SQUARE-CIRCLE pair in the 27 blocks was significant $[F(1,12)=15.90, p<.01]$. This finding replicates the results of Experiments 2 and 3. Other outcomes of the ANOVA did not qualify the interpretation of the predicted interaction.

The mean proportions of errors in Experiment $4 \mathrm{~A}$ for the 27 and all-forms blocks were .044 and $.017(p>.10$,
Wilcoxon signed ranks test). The corresponding Experiment 4B proportions for the 27 and all-forms blocks were .027 and .033 .

\section{Discussion}

"Different"' responses to the SQUARE-CIRCLE pair were slower on the 27 blocks than on the all-forms blocks. Earlier discussion indicates that this result may mean that memory for the second-category 27 increased the perceived similarity of the SQUARE and CIRCLE stimuli. The error proportions once again argue against the possibility of a criterion shift between the two types of blocks.

"Same" responses to the SQUARE-SQUARE and CIRCLE-CIRCLE pairs were slightly faster on the 27 blocks than on the all-forms blocks. It follows that the slower "different" responses in Experiments 2-4A were not due to the greater difficulty of the 27 blocks, because the same stimuli occurred for both "same" and "different" responding. Therefore, it does not seem that the second-category 27 led to confusion or inhibition or required more time to respond because of the need to switch attention between categories.

"Same" responding on the 27 blocks resulted in a relatively low proportion of errors to the SQUARE-CIRCLE pairs. Another study (King, 1983) also used blocks of trials to place stimuli into memory, but with unidimensional stimuli. The analogous pairs were small-large pairs of disks that immediately (unexpectedly) followed blocks of trials with pairs of more extreme (tiny and huge) disks. The analogous pairs resulted in errors quite frequently, perhaps because their unexpectedness made it difficult to respond more slowly in order to reduce errors. However, the present SQUARE-CIRCLE pairs occurred relatively frequently. Therefore, subjects may have moderated their "same" responding on the 27 blocks in order to reduce errors. This moderation would also explain why "same" responding on the 27 blocks was only slightly faster than "same" responding on the all-forms blocks.

It is not clear why rehearsal of the anchor (i.e., 27) or forgetting should differentially affect "same" and "different" responses. Thus the obtained differential effect opposes the rehearsal and forgetting possibilities.

Criterial variance theories also need to explain how an influence of the anchor on judgmental categories differentially affects "same" and "different" responses. Suppose that the anchor does decrease the number of categories between the two categories that the simultaneously present stimuli contact. Even so, positing that this decrease slows "different" responses and accelerates "same" responses simply substitutes the distance between judgmental categories (i.e., the similarity in their serial positions on the dimension) for perceived similarity.

It can be estimated that the increase in perceived similarity of the SQUARE and CIRCLE stimuli occurred within about $484 \mathrm{msec}$ minus $100 \mathrm{msec}$, or $384 \mathrm{msec}$ from stimulus onset, assuming that it takes about $100 \mathrm{msec}$ to prepare and execute a response. 


\section{EXPERIMENT 5}

In Experiment 5, we sought to obtain evidence for an anchor-range effect that is due to the simultaneous presence of the anchor and more related stimuli as parts of a figural stimulus. The anchor and more related stimuli have occurred successively in almost all prior research.

Unidimensional stimuli were used. Each stimulus consisted of two small disks and one large disk located around the circumference of an imaginery circle. There were two sizes of the large disk. The assumption was that the larger of the two large disks would result in a stronger anchorrange (increase-in-perceived-similarity) effect. Therefore, it was expected that the two small disks would be perceived as more similar when the more extreme large disk (i.e., the anchor) was present. Speeded "same" and "different" responses were used as measures of perceived similarity.

Blocks of trials with the anchor disk stimuli were unexpectedly followed by single trials with the less extreme large disk stimuli and vice versa. It was predicted that the two small disks would be perceived as more similar when the anchor disk was present for both the block and unexpected types of trials. Confirmation of this prediction would indicate that the memory for the anchor disk was not influential, because the unexpected trials with this disk would be preceded by trials with the less extreme large disk.

An influence of the concurrently present anchor would obviously argue against memorial theories of the anchorrange effect.

According to the category-superordinate theory, the extreme disk would increase the strength of a categorysuperordinate for small disks, and the perceived similarity of the small disks would thereby increase. The predicted result would therefore imply that a fast stimulusproduced influence on the superordinate can occur. In contrast, Experiments 2-4 implied that the fast influence on the superordinate was memorial.

According to the distance-density theory, the predicted result would imply that a concurrently present stimulus can quickly alter density.

\section{Method}

Stimuli. Figure 1 shows the eight stimuli of the experiment. Tiny (T), small (S), huge (H), and gigantic (G) disks were used to form these stimuli. They were $3,5,10$, and $21 \mathrm{~mm}$ in diameter, respectively, and they came from either Letraset Sheet 2453 or Mecanorma Sheet $\mathbf{S 4}$.

Each stimulus contained two disks from the set of $\mathrm{T}$ and $\mathrm{S}$ (small) disks and one disk from the set of $\mathrm{H}$ and $\mathrm{G}$ (large) disks. The centers of the three disks of each stimulus were $120^{\circ}$ apart on the circumference of an imaginary circle with a diameter of $11 \mathrm{~mm}$. The leftmost disk was $25^{\circ}$ above the horizontal. The identities of the left, right, and bottom disks of each stimulus are designated in this order.

Experimental design and trials. One group was told to respond only when all three disks were different. A second group was told to respond only when two disks were the same.

Half of both these groups received five sequences of trials, each consisting of a block of trials with intermixed TGS, TGT, STG, and GSS stimuli followed by a single unpredictable THS, THT,

\section{TGS}

THS
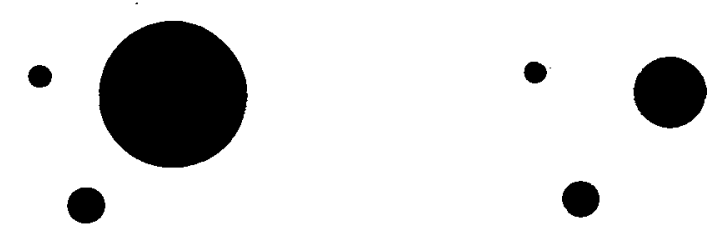

TGT

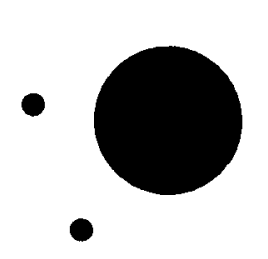

STG
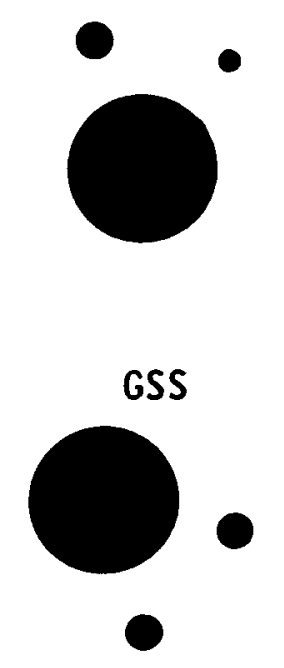

HSS

Figure 1. The stimuli for Experiment 5.

STH, or HSS stimulus. Then, in Stage 2, there were another five sequences of trials, each consisting of a block of trials with intermixed THS, THT, STH, and HSS stimuli followed by a single unpredictable TGS, TGT, STG, or GSS stimulus. The other half of each group received the $G$ and $H$ stimuli in the reverse order; that is, the blocks of the first stage were with the $\mathrm{H}$-disk stimuli, and so on. The stages for the G-blocks-first groups and the H-blocksfirst groups corresponded exactly. For example, each TGT stimulus in Stage 1 for the G-blocks-first group was replaced by a THT stimulus in Stage 1 for the H-blocks-first group. One trial followed the other at an approximately constant rate.

The rest of the trial procedures also followed the earlier study (King, 1983). The number of trials in each block was randomly 
selected from the numbers 5 through 12 . Hence the single trials with the other size of large disk occurred unpredictably. The five blocks of the second stage occurred in a different order. The stimuli in each block were selected randomly, except that the number of consecutive "same" and "different" trials and the proportions of these trials were controlled and there was at least one trial with each stimulus. The unexpected different stimuli occurred after the first, third, and fifth blocks of Stage 1 and after the first, fourth, and fifth blocks of Stage 2. The unexpected same stimuli therefore occurred after the second and fourth blocks of Stage 1 and after the second and third blocks of Stage 2 .

Apparatus, Procedures, and Subjects. An Iconix 6137 tachistoscope was used. One arm contained a warning stimulus, a $7.6 \times 12.7 \mathrm{~cm}$ gray rectangle centered on a black background. The disk stimuli were placed in a second arm. The distance from the stimuli to the cornea was approximately $90 \mathrm{~cm}$.

The experimenter said "Ready" to begin a trial. The durations of the warning stimulus and the interstimulus interval were both $1 \mathrm{sec}$. A stimulus followed, and it terminated either with a response or after $3 \mathrm{sec}$. Stimulus timing and responding were measured in milliseconds. The experimenter provided accuracy and reaction time feedback after each trial.

There were 5 Howard University undergraduates in each of the four groups. Participation satisfied an introductory course requirement.

\section{Results}

Time results. The ANOVA was a 2 (response: "same" and "different") $\times 2$ (order: G blocks first and $\mathrm{H}$ blocks first) $\times 2$ (large disk: $\mathrm{G}$ and $\mathrm{H}) \times 2$ (trial type: block and unexpected) $\times 2$ (stimulus type: large disk to the right and large disk to the left or bottom) factorial. The first two factors were between groups.

The response time for an individual unexpected stimulus was used for the ANOVA. However, both the TGS and THS stimuli occurred on two of the unexpected trials of a stage, and therefore the mean of the two response times to each of these stimuli was used. The block scores were, with one exception, obtained from only those blocks that preceded a to-be-responded-to unexpected stimulus. Only the times to the analogous stimuli were used. For example, when the TGS stimulus was unexpected, the corresponding block score equaled the mean of the response times to the THS stimuli of the immediately preceding block. However, times to the first stimulus of a block were disregarded, due to the possibility that they might be influenced by the switch from the immediately preceding unexpected stimulus. Because both the TGS and THS stimuli occurred on two of the unexpected trials of a stage, there were two corresponding block scores for each stage, and hence the mean of the two scores was used. The ex- ception referred to above was that Block 2 rather than Block 1 was used for the TGS and THS stimuli of the first stage. The slow responses on the first few trials of the experiment were thereby avoided.

The important result was the response $\times$ large disk interaction $[F(1,16)=19.78, p<.001]$. Plannedcomparison tests revealed that the G-disk stimuli resulted in significantly slower "different" responses and significantly faster "same" responses than the H-disk stimuli $[F(1,16)=11.99, p<.01$ and $F(1,16)=8.04, p<.05$, respectively]. In addition, the magnitude of both these outcomes was greater for the unexpected trials, as indicated by the response $\times$ large disk $\times$ trial type interaction $[F(1,16)=5.99, p<.05]$. Table 1 shows the three-way interaction means. There were no other significant outcomes.

Error results. Error proportions were calculated for the eight conditions that result from the factorial combination of two responses, two large disks, and two trial types, and these proportions are also indicated in Table 1.

For "different" responding, the error proportions (false "differents") were lower for the G-disk stimuli than for the H-disk stimuli ( $p<.05$, one-tailed, Wilcoxon signed ranks test, for both the unexpected and block comparisons). However, for "same" responding, the error proportions (false "sames") were higher for the G-disk stimuli than for the H-disk stimuli $(p<.10$, one-tailed, Wilcoxon signed ranks test, for both the unexpected and block comparisons).

\section{Discussion}

The G-disk stimuli resulted in faster "same" and slower "different" response times than the H-disk stimuli. As before, fast "same" and slow "different" responses are taken to indicate high perceived similarity. Thus, the two small disks were perceived as more similar when the $G$ (gigantic, or anchor) disk occurred. This inference supports the conclusion that the anchor disk increased the perceived similarity of the two small disks, in line with the increase-in-perceived-similarity interpretation of the anchor-range effect.

Furthermore, the $\mathrm{G}$ (anchor) disk was influential when it and the two small disks were simultaneously present. The supporting evidence for this influence is that the increase-in-perceived-similarity result occurred for the unexpected as well as for the block trials. The $G$ disk was not in memory on the unexpected trials, because an un-

Table 1

Response Times and Errors for Experiment 5

\begin{tabular}{lccccc}
\hline & \multicolumn{2}{c}{ G (Anchor) Disk } & & \multicolumn{2}{c}{ H Disk } \\
\cline { 2 - 3 } \cline { 5 - 6 } Measures & $\begin{array}{c}\text { Block } \\
\text { Trials }\end{array}$ & $\begin{array}{c}\text { Unexpected } \\
\text { Trials }\end{array}$ & & $\begin{array}{c}\text { Block } \\
\text { Trials }\end{array}$ & $\begin{array}{c}\text { Unexpected } \\
\text { Trials }\end{array}$ \\
\hline "Different" Response Times & 613 & 737 & & 565 & 531 \\
"Same" Response Times & 494 & 460 & & 563 & 599 \\
False "Different" Errors & .04 & .05 & & .13 & .35 \\
False "Same" Errors & .04 & .11 & & .02 & .04 \\
\hline
\end{tabular}


expected G-disk stimulus was immediately preceded by a block of trials with $\mathrm{H}$-disk stimuli. Therefore, the $\mathrm{G}$ disk must have been influential when it was actually present. In contrast, earlier research (King, 1983), despite the use of similar procedures, pointed to a memorial influence of the extreme stimuli.

The error results also support the conclusion that the $G$ disk increased the perceived similarity of the two small disks. Recall that the G-disk stimuli resulted in fewer errors when "different" responses were made and more errors when "same" responses were made. Following King (1983), the interpretation is that the G disk increased the perceived similarity of the $T$ and $T$ and $S$ and $S$ disks and thereby reduced the probability of falsely identifying them as different (i.e., more similar stimuli should be less likely to be incorrectly identified as different). Also, the $G$ disk increased the perceived similarity of the $T$ and $S$ disks and thereby raised the probability of falsely identifying them as the same (i.e., more similar stimuli should be more likely to be incorrectly identified as the same).

The error results also imply that the $G$ disk was influential when it occurred, rather than when it was in memory. This is because the G-disk stimuli resulted in the same pattern of errors for both the unexpected and block trials.

The possibility that different speed-accuracy criteria were in effect for the G-disk and $\mathrm{H}$-disk blocks does not explain why "same" and "different" responding were affected in opposite directions. Furthermore, the block and unexpected trials resulted in much the same outcome, even though the decision criterion for the $\mathrm{H}$-disk blocks would have been in effect for the unexpected G-disk stimuli (because they followed immediately) and vice versa.

Similarity could have been influential in another way, and yet it was not, which indirectly supports the anchorrange interpretation. The anchor $(\mathrm{G})$ disk was less similar to the two small disks than the less extreme $(\mathrm{H})$ disk was. As previously indicated, less similar stimuli result in faster "different" responses. Thus, it could have been expected (incorrectly) that the G-disk stimuli would have resulted in faster "different" responses than the H-disk stimuli.

According to another similarity explanation, the $\mathrm{H}$ disk was more similar than the $\mathrm{G}$ disk to the two small disks, and therefore the $\mathbf{H}$ disk was more likely to be substituted for (confused with) one of these small disks. This tendency would account for the error results (e.g., substitution of the $\mathrm{H}$ disk for one of the same small disks would increase false "different" responses). In addition, the disparity between the false "different" and false "same" error proportions was greater for the H-disk stimuli, which suggests that the $\mathrm{H}$ disk was causal. However, this explanation also predicts (incorrectly) that the H-disk stimuli would have resulted in both slower "different" and slower "same" responses than the G-disk stimuli, because more similar stimuli should take longer to discriminate among. In support, a third more similar (although not simultaneously present) stimulus slowed down both "same" and "different" responses (Crist, 1981).

The G-disk stimuli resulted in a mean "same" response time of $477 \mathrm{msec}$. Consequently, the $G$ disk may have increased the perceived similarity of the two simultaneously present small disks within roughly $377 \mathrm{msec}$ of stimulus onset.

Egeth, Avant, and Bevan (1968) found that similarly colored chips were judged to be more similar as the color of the background became more extreme. Therefore, Egeth et al. may have also obtained the anchor-range effect with a simultaneously present anchor. However, the similarly colored chips surely contrasted more with the background as it grew more extreme, and this joint increase in contrast could have augmented judged similarity.

The present experiment clearly calls into question the rehearsal and forgetting theories of the anchor-range effect and also the memorially based criterial variance theory of Durlach and Braida (1969). This is because the anchor (G disk) was influential when it was present, rather than in memory.

The category-superordinate theory assumes that the $G$ (anchor) disk increased the strength of the category of small disks. Thus, category strength would have to have been quickly influenced by an immediately present stimulus.

The density in the region of the little disks should be less with the $\mathrm{G}$ (anchor) disk than with the $\mathrm{H}$ disk. Therefore, the finding that the $\mathrm{G}$ disk was influential in the trials on which it occurred implies that a concurrent stimulus can quickly alter density.

\section{GENERAL DISCUSSION}

We conclude that memory for a stimulus from one category increased the perceived similarity of two stimuli from a second category. We also conclude that a concurrently present extreme third stimulus increased the perceived similarity of two more related unidimensional stimuli.

The second-category stimuli and the concurrent extreme disk are analogous to conventional anchors. The samecategory stimuli and the simultaneously present more similar disks correspond to conventional more related unidimensional stimuli. The two increase-in-perceivedsimilarity outcomes were therefore interpreted as anchorrange effects.

The results call into question memorial theories of the anchor-range effect. The rehearsal and forgetting possibilities seem unlikely, because the relevant stimuli were simultaneously present (Experiment 1), the task was to discriminate between simultaneously present stimuli (Experiments 2-5), and "same" and "different" responses were differentially affected (Experiments 4-5). Furthermore, the extreme disk was influential on the trial on which it occurred (Experiment 5).

Experiments 1-4 call into question the criterial vari- 
ance theories, because they cannot specify the dimensions for stimuli from relatively different categories. Experiments 2-5 required discriminations between simultaneously present stimuli, and yet criterial variance theories presumably posit that judgmental categories are compared. In addition, the assumed influence of the anchor on these categories still does not account for the anchor's ability to differentially affect "same" and "different" responses.

The results more directly back the increase-inperceived-similarity interpretation. First, the similarity rating results were supportive. Second, perceived similarity is known to accelerate "same" responses and to slow down "different" responses, and this opposite-direction outcome was obtained. Third, the speeded response research eliminated the possibility of a slow cognitive postonset influence on the current response. Fourth, the figural stimuli of Experiment 5 were obviously perceptual in nature, in accord with the increase-in-perceivedsimilarity (and hence perceptual) interpretation.

The category-superordinate theory was supported. This support enhances the possibility that a superordinate or correlated factor can quickly influence perceived similarity (Experiments 2-5). It also implies that both a remembered stimulus (Experiments 2-4) and an immediate stimulus (Experiment 5) can quickly influence the strength of a superordinate.

The present research also supports the distance-density theory, indicating that it applies when density would have to be quickly influential (Experiments 2-5) and when it would have to be quickly altered by a concurrently present stimulus (Experiment 5).

The category-superordinate theory links up with indications that perceived similarity also increases with the strength of gestalts (superordinates). Stimuli tend to be perceived as more similar in color when they are parts of the same gestalt (e.g., Day, 1983). In addition, objects are perceived as closer together, that is, in more similar spatial positions, when they belong to the same gestalt (Coren \& Girgus, 1980). In contrast, the distance-density theory is contradicted by the finding that an increase in rated similarity is produced by more as well as less similar stimuli (King \& Atef-vahid, 1984).

In conclusion, the anchor-range effect can occur when the anchor is a second-category stimulus and when it is simultaneously present as part of a figural stimulus. In addition, the arguments against the rehearsal, forgetting, and criterial variance theories, the measurement of similarity with "same" and "different" responses, and the use of figural stimuli all imply that an anchor increases the perceived similarity of more similar stimuli.

\section{REFERENCES}

Bevan, W., \& Pritchard, J. F. (1963). The anchor effect and the problem of relevance in the judgment of shape. Journal of General Psychology, 69, 147-161.

COREN, S., \& GiRgus, J. S. (1980). Principles of perceptual organization and spatial distortion: The Gestalt illusions. Journal of Experimental Psychology: Human Perception \& Performance, 6, 404-412.

CRIST, W. B. (1981). Matching performance and the similarity structure of the stimulus set. Journal of Experimental Psychology: General, 110, 269-296.

DAY, R. H. (1983). Neon color spreading, partially delineated borders, and the formation of illusory contours. Perception \& Psychophysics, 34, 488-490.

DiLollo, V., \& KirKHAM, R. (1969). Judgmental contrast effects in relation to range of stimulus values. Journal of Experimental Psychology, 81, 421-427.

Durlach, N. I., \& Braida, L. D. (1969). Intensity perception: I. Preliminary theory of intensity resolution. Journal of the Acoustical Society of America, 46, 372-382.

Egeth, H., Avant, L. L., \& Bevan, W. (1968). Does context influence the shape of a perceptual scale? Perception \& Psychophysics, 4, 54-56.

ERIKSEN, C. W., \& HAKE, H. W. (1957). Anchor effects in absolute judgments. Journal of Experimental Psychology, 53, 132-138.

ERIKSEN, C. W. O'HARA, W. P., \& ERIKSEN, B. (1982). Response competition effects in same-different judgments. Perception \& Psychophysics, 32, 261-270.

Gravetter, F., \& Lockhead, G. R. (1973). Criterial range as a frame of reference for stimulus judgment. Psychological Review, 80, 203-216.

Hawkins, H. L., \& Shigley, R. H. (1972). Irrelevant information and processing mode in speeded discrimination. Journal of Experimental Psychology, 96, 389-395.

King, D. L. (1983). Anchor research: Evidence for an increase in the perceived similarity of stimuli obtained with a speeded response paradigm. Journal of Experimental Psychology: Human Perception \& Performance, 9, 474-486.

King, D. L., \& ATEF-VAHID, M. (1984, April). Anchor effect and perceptual grouping. Paper presented at the meeting of the Eastern Psychological Association, Baltimore, MD.

KRUEGER, L. E. (1973). Effect of irrelevant surrounding material on speed of same-different judgment of two adjacent letters. Journal of Experimental Psychology, 98, 252-259.

KRUMHANSL, C. L. (1978). Concerning the applicability of geometric models to similarity data: The interrelationship between similarity and spatial density. Psychological Review, 85, 445-463.

Lockhead, G. R. (1972). Processing dimensional stimuli: A note. Psychological Review, 79, 410-419.

PollaCK, I. (1952). The information of elementary auditory displays. Journal of the Acoustical Society of America, 24, 745-749.

Proctor, R. W. (1981). A unified theory for matching-task phenomena. Psychological Review, 88, 291-326.

SHEPARD, R. N. (1964). Attention and the metric structure of the stimulus space. Journal of Mathematical Psychology, 1, 54-87.

SIEGEL, W. (1972). Memory effects in the method of absolute judgment. Journal of Experimental Psychology, 94, 121-134.

SرöвERG, L. (1972). A cognitive theory of similarity. Göteborg Psychological Reports, 2, No. 10.

TORGERSON, W. S. (1958). Theory and methods of scaling. New York: Wiley.

TVErSky, A. (1977). Features of similarity. Psychological Review, 84, 327-352.

Watson, H. D. (1981). The effects of objective and perceived size properties on visual form matching. Journal of Experimental Psychology: General, 110, 547-567.

(Manuscript received January 1, 1985; revision accepted for publication January $6,1986$. 